\title{
Dislipidemia mista e o risco da evolução de doenças cardiovasculares em idosos
}

\author{
Mixed dyslipidemia and the evolution risk of cardiovascular diseases in elders \\ Dislipidemia mixta y riesgo de evolución de enfermedades cardiovasculares en ancianos
}

\author{
Ana Clara Silva Borges \\ ORCID: https://orcid.org/0000-0001-9045-8426 \\ Universidade José do Rosário Vellano, Brasil \\ E-mail: ana.borges@aluno.unifenas.br \\ Bárbara Ferreira Rodrigues \\ ORCID: https://orcid.org/0000-0002-5567-7700 \\ Universidade José do Rosário Vellano, Brasil \\ E-mail: barbara.rodrigues@aluno.unifenas.br \\ Laiane Silva Pires \\ ORCID: https://orcid.org/0000-0002-5886-5387 \\ Universidade José do Rosário Vellano, Brasil \\ E-mail: laiane.pires@aluno.unifenas.br \\ Gabriele Santiago Raimundo Rodrigues \\ ORCID: https://orcid.org/0000-0003-2635-7326 \\ Universidade José do Rosário Vellano, Brasil \\ E-mail: gabriele.rodrigues@aluno.unifenas.br \\ Isabella Cristina Giraldi dos Santos \\ ORCID: https://orcid.org/0000-0002-2267-8862 \\ Universidade José do Rosário Vellano, Brasil \\ E-mail: isabella.giraldi@aluno.unifenas.br \\ Gérsika Bitencourt Santos \\ ORCID: https://orcid.org/0000-0003-0849-2786 \\ Universidade José do Rosário Vellano, Brasil \\ E-mail: gersika.santos@unifenas.br \\ Pedro Ivo Sodré Amaral \\ ORCID: https://orcid.org/0000-0002-2366-2088 \\ Universidade José do Rosário Vellano, Brasil \\ E-mail: pedro.amaral@unifenas.br \\ Roberta Bessa Veloso \\ ORCID: https://orcid.org/0000-0003-4794-5872 \\ Universidade José do Rosário Vellano, Brasil \\ E-mail: bessaveloso@yahoo.com.br
}

\section{Resumo}

A dislipidemia é caracterizada como um distúrbio que causa alteração nos níveis séricos de lipídios do organismo (aumento plasmático de triglicerídeos, LDL-c e colesterol ou redução nos níveis de HDL-c), que é um dos fatores responsáveis por aumentar o risco cardiovascular. Diante disso, a presente revisão de literatura teve como objetivo avaliar a correlação entre a ocorrência de dislipidemias mistas e o risco de evolução para doenças cardiovasculares em idosos, bem como as suas principais causas comportamentais. A busca foi feita em bases científicas, como SciELO, PubMed, Google Scholar e Lilacs. Do total de artigos encontrados (585.558 artigos), foram selecionados 23 para compor esta revisão de literatura. Conclui-se que a dislipidemia mista deixa os idosos mais propensos a apresentarem eventos cardiovasculares, ou seja, existe relação entre esses dois fatores, os quais são causados principalmente pelos maus hábitos de vida, sendo eles obesidade, sedentarismo, alimentação inadequada, tabagismo e ingestão de bebidas alcoólicas. Além disso, a doença está associada principalmente ao gênero feminino.

Palavras-chave: Metabolismo de lipídeos; Fatores de risco; Fenômenos fisiológicos cardiovasculares.

\begin{abstract}
Dyslipidemia causes changes in serum lipid levels (increases plasma triglycerides, LDL, and cholesterol or decreases HDL levels), which is one of the factors responsible for increased cardiovascular risk. In this sense, the present review aimed to assess the relationship between the occurrence of mixed dyslipidemia and the evolution risk of cardiovascular disease in elders, as well as its main behavioral causes. The bibliographic search was made on scientific bases such as SciELO, PubMed, Google Scholar and Lilacs. From the total papers (585,558 papers), twentythree were selected to compose this literature review. It has been observed that mixed dyslipidemia increases the likelihood of cardiovascular effects in elder, i.e., there is a straight relationship between these two factors, which are mainly caused by unhealthy habits, such as obesity, sedentary lifestyle, unhealthy diet, smoking, and consumption of alcoholic beverages. In addition, the disease is mainly associated with feminine gender.
\end{abstract}


Keywords: Lipid metabolism; Risk factors; Cardiovascular physiological phenomena.

\section{Resumen}

La dislipidemia se caracteriza por ser un trastorno que provoca cambios en los niveles séricos de lípidos en el organismo (aumento plasmático de triglicéridos, LDL y colesterol o reducción de los niveles de HDL), que es uno de los factores responsables del aumento del riesgo cardiovascular. Por ello, la presente revisión de la literatura tuvo como objetivo evaluar la correlación entre la aparición de dislipidemias mixtas y el riesgo de progresión a enfermedades cardiovasculares en los ancianos, así como sus principales causas de comportamiento. La búsqueda se realizó sobre bases científicas, como SciELO, PubMed, Google Scholar y Lilacs. De la cantidad total de artículos encontrados (585.558 artículos), 23 fueron selecionados para componer esta revisión de literatura. Se concluye que la dislipidemia mixta hace que los ancianos sean más propensos a presentar eventos cardiovasculares, es decir, existe una relación entre estos dos factores, los cuales son causados principalmente por malos hábitos de vida, que son obesidad, estilo de vida sedentario, dieta inadecuada y consumo de tabaco y alcohol. Además, la enfermedad se asocia principalmente al género femenino.

Palabras clave: Metabolismo de los lípidos; Factores de riesgo; Fenómenos fisiológicos cardiovasculares.

\section{Introdução}

A população idosa encontra-se em crescimento constante e, consequentemente, o número de enfermidades também cresce a cada ano, destacando-se os eventos cardiovasculares, pois são a maior causa de morte nessa faixa etária (Borim, Francisco \& Neri, 2017). Dentre as causas relacionadas às doenças cardíacas, a dislipidemia é classificada como uma das principais (Prince et al., 2014).

A dislipidemia é caracterizada por alterações nos níveis séricos de lipídios, ou seja, aumento plasmático de triglicerídeos, lipoproteína de baixa densidade (LDL-c) e colesterol ou redução nos níveis de lipoproteína de alta densidade (HDL-c) (Kopin \& Lowenstein, 2017). As alterações no colesterol total e nas frações lipídicas podem ser isoladas ou mistas. Segundo a Atualização da Diretriz Brasileira de Dislipidemias e Prevenção da Aterosclerose (2017), a hiperlipidemia mista é caracterizada pelo aumento simultâneo de LDL-c e de triglicerídeos.

Todavia, fato relevante na hiperlipidemia do idoso é que raramente ocorrem grandes elevações de colesterol, de triglicérides e de LDL-c, sendo, em geral, elevações discretas a moderadas. Sendo assim, as dislipidemias diagnosticadas nessa idade não são primárias, ou seja, de caráter genético, mas sim secundárias a diversos fatores, como hipotireoidismo, diabetes mellitus, intolerância à glicose, obesidade, síndrome nefrótica e uso de medicamentos, como diuréticos tiazídicos e bloqueadores beta-adrenérgicos não seletivos (Atualização da Diretriz Brasileira de Dislipidemias e da Prevenção da Aterosclerose, 2017). Ademais, estudos conduzidos com a população idosa mostraram que os níveis socioeconômicos e a alimentação são fatores que corroboram para eventos cardiovasculares (Martins et al., 2017).

São poucos os estudos que possuem como objetivo a análise da situação de saúde da população idosa do Brasil. Nesse sentido, é de extrema importância que tais estudos sejam realizados para que haja melhorias na prevenção e na atuação dos profissionais de saúde (Olubamwo et al., 2018). Com isso, é possível reduzir os índices de morbimortalidade, visto que as doenças cardiovasculares são as principais causas de morte no mundo (Azzi, Vale \& Silva, 2018).

Nessa perspectiva, o objetivo do presente trabalho é avaliar a relação entre a dislipidemia mista e a evolução de doenças cardiovasculares em idosos, bem como as suas principais causas comportamentais modificáveis.

\section{Metodologia}

Este estudo é uma revisão de literatura (Pereira et al., 2018), que apresenta como tema a relação da dislipidemia mista e o risco da evolução de doenças cardiovasculares em pessoas idosas. A identificação dos artigos foi feita por meio de buscas em bases científicas, como Scientific Electronic Library Online (SciELO), o PubMed, o Google Scholar e a Literatura LatinoAmericana e do Caribe em Ciências da Saúde (Lilacs), selecionando os trabalhos publicados entre os anos de 2000 a 2020. Para a busca dos artigos, foram utilizados os termos chaves padronizados pelos Descritores em Ciências da Saúde (DeCS): 
idosos e doenças; idosos e cardiopatias; idosos e cardiovascular; idosos e lipídios; doenças cardiovasculares e fatores de risco; lipídios e cardiopatias; idosos e obesidade; lipídios e obesidade; elderly e cholesterol; elderly e obesity; ancianos e colesterol.

A seleção dos artigos baseou-se na conformidade dos limites dos assuntos aos objetivos deste trabalho. Como critérios de inclusão, foram considerados tanto artigos científicos publicados em revistas quanto anais publicados em congresso e que utilizaram estudos longitudinais; estudos controlados randomizados; estudos observacionais descritivos de corte transversal; estudos de prevalência; estudos descritivos; estudos transversais; estudos transversais de base populacional; estudos de corte transversal e caráter descritivo; estudos retrospectivos e descritivos; estudos retrospectivo, descritivo e analítico; estudos observacionais descritivos com corte transversal temporalmente retrospectivo; estudos de prevalência com delineamento transversal e observacional; estudos quantitativos, transversais e descritivos; artigos de revisão; estudos de atualização; estudos transversais, descritivos e analíticos; estudos transversais, observacionais, analíticos e de base populacional; estudos analíticos; e estudos quantitativos e que tenham sido publicados em português, inglês ou espanhol. Foram excluídos os estudos publicados em outros idiomas, que não sejam português, inglês ou espanhol, e os trabalhos de conclusão de curso, sejam eles monografia, dissertação ou tese.

\section{Resultados}

Utilizando os descritores supracitados, foram obtidos um total de 585.558 resultados, sendo que foram analisados 76 resumos de acordo com o título e o ano de publicação. A partir disso, 38 estudos foram selecionados para a análise, uma vez que relacionavam o fator idade com índices antropométricos, risco cardiovascular, níveis lipídicos, comorbidades ou estilo de vida. Para a seleção, os autores utilizaram os mesmos critérios de inclusão e exclusão. Por fim, foram selecionados 23 artigos que abordavam sobre dislipidemia mista, dando ênfase aos fatores de risco associados à evolução cardiovascular em idosos (Tabela 1). 
Tabela 1. Distribuição das referências bibliográficas obtidas nas bases de dados Google Scholar, Lilacs, SciELO e PubMed, de acordo com as palavras-chaves selecionadas.

\begin{tabular}{|c|c|c|c|c|c|}
\hline $\begin{array}{l}\text { Bases de } \\
\text { Dados }\end{array}$ & Estratégias de buscas & $\begin{array}{c}\text { Número de } \\
\text { Referências } \\
\text { Obtidas }\end{array}$ & $\begin{array}{c}\text { Resumos } \\
\text { Analisados }\end{array}$ & $\begin{array}{l}\text { Referências } \\
\text { Selecionadas } \\
\text { para Análise }\end{array}$ & $\begin{array}{l}\text { Selecionados } \\
\text { para Revisão }\end{array}$ \\
\hline \multirow{8}{*}{$\begin{array}{l}\text { Google } \\
\text { Scholar }\end{array}$} & Idosos, doenças & 105.000 & 8 & 4 & 2 \\
\hline & Idosos, cardiopatias & 15.400 & 10 & 3 & 2 \\
\hline & Idosos, lipídios & 15.400 & 7 & 5 & 2 \\
\hline & Idosos, cardiovascular & 17.200 & 5 & 4 & 3 \\
\hline & Mortalidade, idosos & 112.000 & 2 & 2 & 2 \\
\hline & $\begin{array}{l}\text { Mortalidade, } \\
\text { cardiovascular }\end{array}$ & 52.200 & 1 & 1 & 1 \\
\hline & $\begin{array}{l}\text { Fatores de risco, } \\
\text { cardiovasculares }\end{array}$ & 77.000 & 1 & 1 & 1 \\
\hline & Idosos, obesidade & 55.800 & 1 & 1 & 1 \\
\hline \multirow[t]{3}{*}{ Lilacs } & $\begin{array}{c}\text { Doenças } \\
\text { cardiovasculares, fatores } \\
\text { de risco }\end{array}$ & 3.445 & 5 & 3 & 1 \\
\hline & Idosos, lipídios & 176 & 3 & 2 & 2 \\
\hline & Lipídios, cardiopatias & 17 & 2 & 1 & 0 \\
\hline \multirow[t]{4}{*}{ SciELO } & Idosos, obesidade & 238 & 4 & 1 & 1 \\
\hline & Elderly, cholesterol & 153 & 3 & 2 & 2 \\
\hline & Lipídios, obesidade & 67 & 2 & 1 & 1 \\
\hline & Ancianos, colesterol & 22 & 2 & 1 & 1 \\
\hline \multirow[t]{3}{*}{ PubMed } & Elderly, obesity & 131.369 & 12 & 3 & 1 \\
\hline & $\begin{array}{c}\text { Doenças } \\
\text { cardiovasculares, fatores } \\
\text { de risco }\end{array}$ & 26 & 2 & 1 & 0 \\
\hline & Idosos, doenças & 45 & 6 & 2 & 0 \\
\hline Total & - & 585.558 & 76 & 38 & 23 \\
\hline
\end{tabular}

Fonte: Autores (2021).

Os artigos selecionados estão demonstrados na Tabela 2. Foram destacados os autores, o ano de publicação, a metodologia utilizada, os objetivos e os principais achados dos artigos que se enquadraram ao tema da presente revisão. 
Tabela 2. Distribuição das referências inclusas na revisão de literatura, de acordo com o autor e o ano de publicação, o tipo de estudo, os objetivos e os principais achados.

\begin{tabular}{|c|c|c|c|}
\hline $\mathrm{N}^{\mathrm{o}}$ & Autores (Ano) & Tipo de Estudo & Objetivos \\
\hline 1 & $\begin{array}{l}\text { Zaslavsky \& Gus } \\
\qquad(2002)\end{array}$ & Uma atualização. & $\begin{array}{l}\text { Este estudo teve como objetivo relatar as } \\
\text { alterações biopsicossociais que } \\
\text { acontecem com o envelhecimento, } \\
\text { analisando a doença cardíaca, as } \\
\text { comorbidades, a prevenção de quedas, a } \\
\text { percepção sensorial, a dieta e a atividade } \\
\text { física, a imunização, a sexualidade e a } \\
\text { incontinência urinária, o estado mental e } \\
\text { os aspectos sociais no paciente idoso. }\end{array}$ \\
\hline 2 & $\begin{array}{c}\text { Nagatsuyu, et al. } \\
\text { (2009) }\end{array}$ & Estudo de prevalência. & $\begin{array}{lll}\text { O objetivo foi avaliar a possível } \\
\text { associação entre a medida da } \\
\text { circunferência abdominal (CA) e os } \\
\text { níveis de lipídios no sangue. }\end{array}$ \\
\hline 3 & Schatz, et al. (2001) & Estudo de coorte. & $\begin{array}{l}\text { O objetivo foi investigar as discrepâncias } \\
\text { entre colesterol sérico e todas as causas } \\
\text { de mortalidade em idosos. }\end{array}$ \\
\hline 4 & $\begin{array}{l}\text { Fontana, et al. } \\
\qquad(2013)\end{array}$ & Estudo de prevalência. & $\begin{array}{l}\text { Seu objetivo foi avaliar como a interação } \\
\text { entre esses biomarcadores relacionados } \\
\text { ao envelhecimento afeta a fragilidade em } \\
\text { idosos hospitalizados. }\end{array}$ \\
\hline 5 & $\begin{array}{l}\text { Lotufo, et al. } \\
\qquad(2017)\end{array}$ & Estudo descritivo. & $\begin{array}{l}\text { Tal estudo visou avaliar a prevalência de } \\
\text { diagnóstico médico de colesterol alto } \\
\text { autorreferido na população adulta } \\
\text { brasileira, utilizando-se dos dados da } \\
\text { Pesquisa Nacional de Saúde de } 2013\end{array}$ \\
\hline 6 & Gomez (2005) & $\begin{array}{lr}\text { Estudo observacional } \\
\text { descritivo com } & \text { corte } \\
\text { transversal } & \\
\text { temporalmente } & \\
\text { retrospectivo, } & \text { com } \\
\text { amostragem } & \text { não } \\
\text { probabilística de } & \text { casos }\end{array}$ & $\begin{array}{l}\text { Tal estudo teve como objetivo } \\
\text { estabelecer a relação das dislipidemias } \\
\text { em pacientes maiores de } 60 \text { anos, } \\
\text { relacionar a associação de dislipidemias } \\
\text { com outras entidades nosológicas e } \\
\text { determinar a frequência das frações do } \\
\text { colesterol de acordo com o sexo. }\end{array}$ \\
\hline
\end{tabular}

Principais Achados

Concluiu-se que é necessário suporte aos idosos, utilizando apropriados protocolos preventivos para as comorbidades que os acometem, grupos multidisciplinares e estratégias individualizadas para determinadas especificidades, idades e probabilidades de vida, como também preferências individuais e desejos.

Como principais achados foi encontrada a não relação entre o colesterol total e a CA; a não relação entre o HDL e a CA; e a associação entre triglicerídeos e CA nas mulheres e não nos homens, concluindo que, em idosos, o risco cardiovascular advindo da obesidade centrípeta não tem na sua etiologia, exclusivamente, o aumento de lipídios no sangue.

Como resultado, concluiu-se que o colesterol médio caiu significativamente com o aumento da idade e apenas o grupo com colesterol baixo em ambos os exames tiveram uma associação significativa com a mortalidade.

Concluiu-se que existe relação entre esses fatores biológicos e a mortalidade, ou seja, foi avaliado o prognóstico da mortalidade por meio dos biomarcadores.

principal achado foi que, dos 60.202 participantes analisados, $14,3 \%$ nunca tiveram o colesterol ou os triglicerídeos dosados; o diagnóstico médico de colesterol alto foi de $12,5 \%$ maior nas mulheres do que nos homens; e a frequência de diagnóstico médico de colesterol alto foi maior nos indivíduos de até 59 anos, brancos ou de origem asiática, de maior escolaridade e entre os moradores das regiões Sul e Sudeste do Brasil.

Conclui-se que as dislipidemias em idosos são mais frequentes entre as mulheres, que a idade mais afetada está entre 60 e 70 anos e que a hipertensão arterial sistêmica (HAS) é a doença mais frequentemente associada às dislipidemias. 


\begin{tabular}{|c|c|c|c|}
\hline & & consecutivos. & \\
\hline 7 & $\begin{array}{l}\text { Pereira, et al. } \\
\quad(2008)\end{array}$ & $\begin{array}{l}\text { Estudo transversal de } \\
\text { base populacional. }\end{array}$ & $\begin{array}{l}\text { Tal estudo objetivou estimar prevalência } \\
\text { e aglomeração de fatores de risco e } \\
\text { investigar associação com doença } \\
\text { isquêmica do coração (DIC) em idosos. }\end{array}$ \\
\hline 8 & Souza, et al. (2020) & $\begin{array}{lrr}\text { Estudo } & \text { de } & \text { corte } \\
\text { transversal } & \text { e } & \text { caráter } \\
\text { descritivo. } & & \\
& & \end{array}$ & $\begin{array}{l}\text { Tal estudo teve como objetivo avaliar a } \\
\text { correlação entre os indicadores de RCV } \\
\text { (circunferência da cintura (CC), razão } \\
\text { cintura estatura (RCE) e área de gordura } \\
\text { visceral (AGV)) em idosos } \\
\text { acompanhados por uma unidade de saúde } \\
\text { da Estratégia de Saúde da Família (ESF) } \\
\text { na cidade de Barreiras (BA). }\end{array}$ \\
\hline 9 & $\begin{array}{l}\text { Magalhães, } \\
\text { Amorim \& Rezende } \\
\text { (2018) }\end{array}$ & Estudo de prevalência. & $\begin{array}{l}\text { Tal estudo teve como objetivo analisar as } \\
\text { medidas de ocorrências mais importantes } \\
\text { como prevalência de HAS no Brasil e no } \\
\text { mundo. }\end{array}$ \\
\hline 10 & $\begin{array}{l}\text { Moretti, et al. } \\
\quad(2009)\end{array}$ & $\begin{array}{l}\text { Estudo retrospectivo e } \\
\text { descritivo. }\end{array}$ & $\begin{array}{l}\text { Tal estudo teve como objetivo verificar a } \\
\text { relação entre estado nutricional e } \\
\text { prevalência de dislipidemias numa } \\
\text { população de idosos atendidos no } \\
\text { município de Criciúma (SC), }\end{array}$ \\
\hline 11 & $\begin{array}{l}\text { Almeida, et al. } \\
\text { (2016) }\end{array}$ & $\begin{array}{l}\text { Estudo retrospectivo, } \\
\text { descritivo e analítico. }\end{array}$ & $\begin{array}{l}\text { Tal estudo teve como objetivo analisar o } \\
\text { perfil lipídico e o RCV de pacientes } \\
\text { idosos atendidos no Centro de } \\
\text { Hematologia e Laboratório de Análises } \\
\text { Clínicas LTDA - Hemoclin. }\end{array}$ \\
\hline \multirow[t]{2}{*}{12} & Souza, et al. (2016) & Estudo observacional. & $\begin{array}{l}\text { Tal trabalho visou avaliar o perfil } \\
\text { lipídico dos pacientes com idade entre } 60 \\
\text { a } 79 \text { anos atendidos no Laboratório } \\
\text { Clínico da Pontifícia Universidade } \\
\text { Católica de Goiás em Goiânia. }\end{array}$ \\
\hline & & Estudo de prevalência. & $\begin{array}{l}\text { Tal estudo visou observar a prevalência } \\
\text { da obesidade e a relação entre vários }\end{array}$ \\
\hline
\end{tabular}

da obesidade e a relação entre vários
Como principal achado concluiu-se que os idosos que possuem quatro ou mais fatores de risco cardiovascular (RCV) apresentaram probabilidade quatro vezes maior de apresentar DIC do que aqueles que apresentaram apenas um ou nenhum fator.

Como resultado, constatou-se correlações positivas e

estatisticamente significantes entre os indicadores de RCV.

Concluiu-se que é alta a prevalência de HAS, a mortalidade causada por ela vem crescendo e dentre muitas causas pode perceber os crescentes fatores de RCV e o subfinanciamento do Sistema Único de Saúde (SUS).

Obteve-se como principal achado a maior prevalência das

dislipidemias em idosos obesos e do sexo feminino.

Como resultado, constatou-se que o RCV dos idosos analisados, feito através da razão LDL-c e HDL-c, encontrou-se baixo, variando entre baixíssimo e moderado. De um modo geral, os idosos apresentaram colesterol total, LDL-c, colesterol não-HDL e triglicerídeos dentro da normalidade, porém uma quantidade considerável de mulheres apresentou LDL-c e colesterol não-HDL alto ou muito alto e os níveis de HDL-c mantiveram-se baixos em ambos os sexos.

Os principais achados foram que, dos 158 indivíduos analisados, a maioria era do sexo feminino, 54\% apresentavam HDL-c baixo, $32 \%$ foram classificados com hipertrigliceridemia isolada, $9 \% \mathrm{com}$ hipercolesterolemia isolada e 5\% com hiperlipidemia mista, sendo que as dislipidemias foram mais frequentes entre as mulheres. Além disso, as alterações de HDL-c foram maiores nas mulheres do que nos homens devido a fatores secundários, como menopausa e reposição hormonal.

Como resultado, concluiu-se que a prevalência da obesidade entre os dosos taiwaneses foi de $16,9 \%$, sendo mais comum entre as 


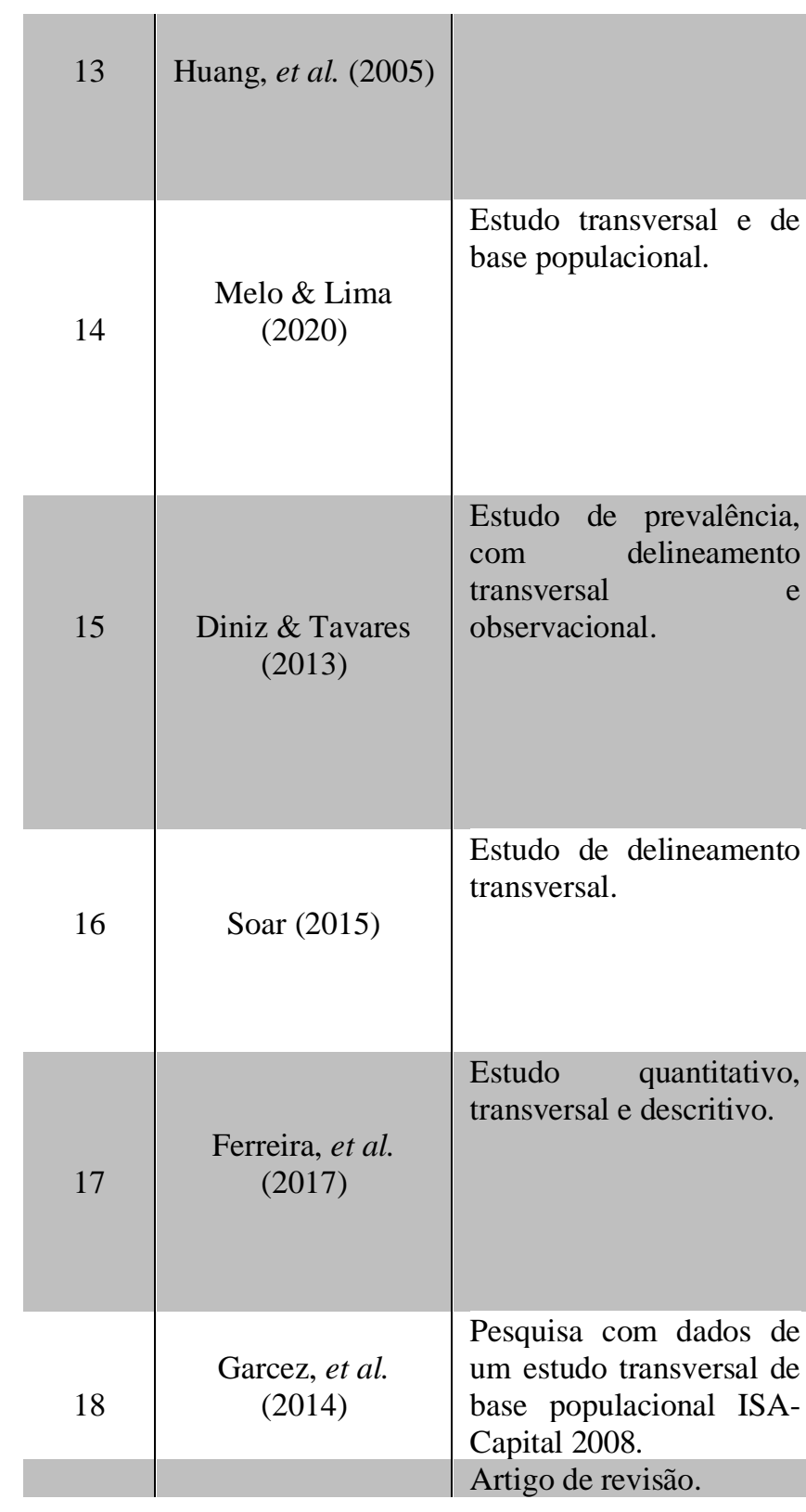

índices antropométricos (Índice de Massa Corporal (IMC), CC e relação cintura-quadril (RCQ)) e fatores de risco para doenças cardiovasculares em idosos.

Tal estudo visou buscar associação entre as multimorbidades mais frequentes em idosos no Brasil e variáveis socioeconômicas e de estilo de vida.

Tal estudo teve como objetivo descrever as características sociodemográficas dos idosos, identificar a prevalência dos fatores de risco para doenças cardiovasculares e compará-los entre os sexos e as faixas etárias.

O objetivo foi descrever as prevalências de fatores de risco para doenças cardiovasculares em idosos não institucionalizados, conforme sexo e grupo etário.

Tal estudo teve como objetivo avaliar as condições de saúde cardiovascular e identificar os fatores de risco modificáveis para doenças cardiovasculares em idosos.

O objetivo foi avaliar o perfil lipídico de residentes no município de São Paulo segundo o estado nutricional. mulheres. Ademais, existe relação entre o RCV e os índices antropométricos, sendo que a $\mathrm{CC}$ se relacionou mais aos fatores de RCV do que o IMC e a RCQ. Porém, nenhum desses índices sozinhos representaram uma boa ferramenta de triagem para o RCV em idosos.

Os resultados obtidos foram que a prevalência de HAS e colesterol alto em idosos está associada ao sexo feminino, aos idosos mais jovens e ao fato de não serem fumantes; a prevalência de HAS e acidente vascular cerebral (AVC) está relacionada ao sexo feminino e aos idosos com ensino fundamental incompleto; e a prevalência de HAS e diabetes está associada aos idosos com ensino fundamental incompleto, não fumantes, não consumidores de bebidas alcoólicas e sedentários.

Como resultado, concluiu que a CA, a HAS e o sedentarismo são os fatores de RCV mais prevalentes, sendo que as mulheres foram mais prevalentes em 6 deles (sedentarismo, CA aumentada, tabagismo, obesidade, HAS e diabetes mellitus), enquanto o alcoolismo foi maior entre os homens.

O resultado obtido foi de que a prevalência de HAS, de hipercolesterolemia, de excesso de peso e de adiposidade abdominal foi, respectivamente, $82,3 \%$ para mulheres e $76,81 \%$ para homens, $43,64 \%$ para mulheres e $25,48 \%$ para homens, $47,4 \%$ para mulheres e $36,12 \%$ para homens e $76,16 \%$ para mulheres e $41,06 \%$ para homens.

Os principais achados foram que $30,1 \%$ são fumantes ativos ou passivos; $80,1 \%$ negaram consumo de bebidas alcoólicas; $32,9 \%$ não se exercitam; 33,3\% encontram-se com sobrepeso; $25,2 \%$ são obesos; $12,6 \%$ apresentam colesterol alto; 34,6\% apresentam glicemia alterada; e a pressão arterial encontra-se alterada em 58,6\% dos idosos na primeira avaliação, $81,8 \%$ na segunda e $74,3 \%$ na terceira.

Como principal achado, foi obtida a confirmação da associação entre dislipidemia e excesso de peso na população da cidade de São Paulo. objetivo foi avaliar se

Como resultado, constatou-se que, diferente do que é comprovado 


\begin{tabular}{|c|c|c|c|c|}
\hline 19 & Santos, et al. (2013) & & $\begin{array}{l}\text { envelhecimento está associado à } \\
\text { obesidade. }\end{array}$ & $\begin{array}{l}\text { em jovens, o sobrepeso em idosos não aumento o risco de } \\
\text { mortalidade, sendo denominado um paradoxo da obesidade. }\end{array}$ \\
\hline 20 & Rocha, et al. (2013) & $\begin{array}{l}\text { Estudo transversal, } \\
\text { descritivo e analítico. }\end{array}$ & $\begin{array}{l}\text { O objetivo foi verificar a correlação entre } \\
\text { os indicadores antropométricos de } \\
\text { obesidade abdominal e lipídios séricos } \\
\text { em idosos. }\end{array}$ & $\begin{array}{l}\text { Como resultado, concluiu que não houve correlações significativas } \\
\text { entre as variáveis para o sexo masculino, porém entre as mulheres a } \\
\text { correlação foi significativa, concluindo que apenas a RCQ e a CC } \\
\text { influenciaram significativamente a variabilidade dos níveis de } \\
\text { triglicerídeos e de HDL, respectivamente. }\end{array}$ \\
\hline 21 & $\begin{array}{l}\text { Rezende, } \text { et al. } \\
\text { (2006) }\end{array}$ & $\begin{array}{l}\text { Estudo de corte } \\
\text { transversal. }\end{array}$ & $\begin{array}{l}\text { Tal estudo teve como objetivo } \\
\text { estabelecer a associação entre IMC e CA } \\
\text { com fatores de risco para doenças } \\
\text { cardiovasculares. }\end{array}$ & $\begin{array}{l}\text { Como resultado, concluiu-se que a frequência de fatores de risco } \\
\text { cardiovascular aumentou com a elevação do IMC e da CA. }\end{array}$ \\
\hline 22 & Cruz, et al. (2004) & $\begin{array}{l}\text { Estudo transversal, } \\
\text { observacional, analítico e } \\
\text { de base populacional. }\end{array}$ & $\begin{array}{l}\text { Tal estudo teve como objetivo avaliar a } \\
\text { prevalência de obesidade e sua } \\
\text { associação com fatores de risco e } \\
\text { morbidades cardiovasculares em idosos } \\
\text { longevos (com idade } \geq 80 \text { anos) } \\
\text { residentes em Veranópolis-RS, Brasil. }\end{array}$ & $\begin{array}{l}\text { Como resultado, observou predominância de obesidade entre os } \\
\text { indivíduos analisados e sua associação ao RCV foi influenciada pelo } \\
\text { sexo, enquanto que não houve diferença entre as morbidades nos } \\
\text { idosos obesos e não obesos. }\end{array}$ \\
\hline 23 & $\begin{array}{l}\text { Marte \& Santos } \\
\quad(2007)\end{array}$ & Artigo de revisão. & $\begin{array}{l}\text { Tal estudo teve como objetivo identificar } \\
\text { os principais fatores que estimulam a } \\
\text { progressão da dislipidemia e da HAS, } \\
\text { com enfoque nos mecanismos que agem } \\
\text { na exacerbação do processo } \\
\text { aterosclerótico e de riscos metabólicos. }\end{array}$ & $\begin{array}{l}\text { O principal achado foi que a HAS pode estar associada ou mesmo } \\
\text { fazer parte de um conjunto de fatores de risco metabolicamente } \\
\text { interligados, os quais determinarão a presença futura de } \\
\text { complicações cardiovasculares. Ademais, existe correlação entre o } \\
\text { perfil lipídico e a pressão arterial sistêmica, uma vez que os } \\
\text { mecanismos fisiopatológicos envolvidos na gênese dessas duas } \\
\text { doenças compartilham anormalidades metabólicas comuns, como a } \\
\text { disfunção endotelial. }\end{array}$ \\
\hline
\end{tabular}

Fonte: Autores (2021). 
Dos 23 artigos selecionados para a revisão, 1 estudo era longitudinal; 2 eram do tipo transversal; 1 era estudo de atualização; 4 eram estudos de prevalência; 1 era estudo descritivo; 3 eram estudos transversais de base populacional; 1 era estudo de corte transversal e caráter descritivo; 1 era estudo retrospectivo e descritivo; 1 era estudo retrospectivo, descritivo e analítico; 1 era estudo observacional descritivo com corte transversal temporalmente retrospectivo; 1 era estudo observacional; 1 era estudo de prevalência com delineamento transversal e observacional; 1 era estudo quantitativo, transversal e descritivo; 2 eram artigos de revisão; 1 era estudo transversal, descritivo e analítico; e 1 era estudo transversal, observacional, analítico e de base populacional; sendo que 2 deles abrangem o assunto de doenças cardíacas, 11 relatam sobre os fatores de risco cardiovascular, 2 discutem sobre a mortalidade em idosos e 8 descrevem sobre dislipidemia englobando alguns dos indicadores antropométricos.

Do total de artigos incluídos na discussão desta revisão de literatura, 18 realizaram estudos com indivíduos, classificando-os de acordo com o tamanho da amostra, a idade ou a média de idade e o gênero. Nesse sentido, o estudo de Nagatsuyu et al. (2009) utilizou como amostra de 98 idosos, sendo 58 mulheres e 40 homens, e a média de idade foi de 66,3 anos; Schatz et al. (2001) analisou 3.572 idosos, com idade entre 71 e 93 anos, e o estudo foi feito apenas com homens; Fontana et al. (2013) analisou 592 idosos, sendo 231 homens e 363 mulheres, e média de idade de 78,7 anos; Pereira et al. (2008) analisou 3.142 idosos, sendo 59,4\% mulheres e 40,6\% homens, e a média de idade foi 69,5 anos; Souza et al. (2020) analisou 49 idosos, sendo 67,3\% do sexo feminino, com média de idade de 71,5 anos; Moretti et al. (2009) estudou 99 idosos, com idade $\geq 65$ anos, sendo 65 mulheres e 34 homens; Almeida et al. (2016) analisou 121indivíduos, sendo que 66,1\% eram idosos com idade de 60 anos ou mais; Gomez (2005) estudou 104 idosos, com idade entre 60 e 90 anos, sendo 47 homens e 57 mulheres; Souza et al. (2016) utilizou amostra de 158 idosos, sendo 102 mulheres e 56 homens, com idade entre 60 e 79 anos; Huang et al. (2005) estudou 2.432 idosos e a média de idade foi de 72,8 anos; Melo e Lima (2020) analisaram 5.575 idosos com média de idade de 70,3 anos; Diniz e Tavares (2013) estudaram 134 idosos, sendo 77 mulheres e 57 homens, com média de idade de 70,5 anos; Soar (2015) analisou 955 idosos, sendo 263 homens de 692 mulheres, com 60 anos ou mais; Ferreira et al. (2017) utilizou amostra de 246 idosos, sendo 75,6\% mulheres; Garcez et al. (2014) estudou 719 indivíduos, sendo que 263 eram idosos com idade de 60 anos ou mais; Rocha et al. (2013) analisou 321 idosos, sendo 67,6\% mulheres e a idade média foi 71,04 anos para o gênero feminino e 71,15 anos para o gênero masculino; Rezende et al. (2006) estudou 231 indivíduos, com idade entre 21 e 76 anos, sendo 106 do sexo feminino e 125 do sexo masculino; e Cruz et al. (2004) analisou 196 idosos, sendo 69 homens e 127 mulheres, com idade $\geq 80$ anos.

\section{Discussão}

O número de pessoas com idade de 60 anos ou mais continua aumentando gradativamente. Segundo a Projeção da População, divulgada pelo IBGE em 2018, os idosos, que em 2018 representavam 9,2\% da população (equivalente a 9,2 milhões), irão representar 25,5\% dela (equivalente a 58,2 milhões) em 2060.

As doenças cardíacas são a maior causa de morbimortalidade. A doença coronariana é responsável por 70 a $80 \%$ de mortes, tanto em homens quanto em mulheres. Já a insuficiência cardíaca congestiva, causa mais comum de internação hospitalar, é a principal responsável pela mortalidade e pela morbidade na população idosa (Zaslavsky \& Gus, 2002). Além disso, segundo Nagatsuyu et al. (2009), existe relação entre a dislipidemia, a obesidade e o envelhecimento, que são fatores preocupantes, pois estão associados às doenças cardiovasculares. Segundo esse estudo, houve grande associação entre triglicerídeos e CA, o que indica aumento do RCV em obesos. Entretanto, na separação por gênero essa associação não foi significativa, apesar de que o aumento da mortalidade cardiovascular nos idosos tem forte relação com a diminuição do HDL e o aumento dos triglicerídeos. 
Níveis elevados de colesterol estão relacionados a um maior risco de desenvolver doença arterial coronariana (DAC) em adultos de meia idade e em pacientes no início da terceira idade. Contudo, com o avanço da idade, essa associação pode se inverter, o que é chamado de "paradoxo do colesterol", porém ainda não se sabe a fisiopatologia desse mecanismo. Entretanto, tanto a prática clínica quanto a literatura mostram que jovens apresentam histórico de infarto agudo do miocárdio mesmo possuindo níveis baixos de colesterol, enquanto idosos com colesterol alto vivem bem, sem manifestação da doença (Schatz et al., 2001). A redução nos níveis de colesterol, que acontece com o avanço da idade, pode ser causada pela fragilidade ou presença de comorbidades, como câncer, desnutrição, fragilidade e doenças crônicas que levam, ao mesmo tempo, à redução do colesterol e ao risco aumentado de morte. Portanto, o colesterol mostra-se como um fator de risco mais relevante para a morte por DAC nos indivíduos mais jovens do que nos mais velhos (Fontana et al., 2013).

O colesterol total apresenta estreita relação negativa com o aumento da idade, porém o motivo dessa relação não apresenta uma causa única e possivelmente relaciona-se com as mudanças na ingestão de nutrientes, no peso corpóreo e no estado de saúde (Nagatsuyu et al., 2009). Ademais, de acordo com Lotufo et al. (2017), a frequência de colesterol elevado aumentou, em ambos os sexos, até os 59 anos, de 60 a 74 anos o valor foi constante e de 75 anos ou mais o valor diminuiu, contudo, seu estudo demonstrou que existe prevalência de dislipidemia na amostra da população brasileira analisada, retirada da Pesquisa Nacional de Saúde de 2013, uma vez que 1 em cada 8 indivíduos relataram apresentar níveis elevados de colesterol.

Segundo Gomez (2005), os níveis de colesterol total aumentam aproximadamente $2 \mathrm{mg} / \mathrm{dl}$ por ano durante a idade adulta jovem até os 65 anos e, então, diminui. Até os 50 anos, os homens apresentam maiores níveis desse lipídio do que as mulheres. Todavia, após a menopausa, o efeito protetor do estrógeno não se faz mais presente nas mulheres, o que culmina no aumento do colesterol e no risco de DAC, assim como relata Pereira, Barreto e Passos (2008). Por fim, o risco torna-se igual em ambos os sexos por volta dos 60 anos de idade. É importante destacar também que, nos idosos, são observadas mudanças na distribuição das lipoproteínas devido não só a fatores genéticos e ambientais, mas também à coexistência de doenças e à deterioração da saúde. As lipoproteínas, além dos níveis de colesterol e de triglicerídeos, apresentam um papel de extrema importância na patogênese da doença aterosclerótica (Gomez, 2005).

Pode-se observar que o estudo realizado por Pereira, Barreto e Passos (2008) identificou que há associação do aumento do risco de doenças isquêmicas do coração em idosos devido à dislipidemia e os fatores de riscos detectados foram obesidade, sedentarismo, tabagismo, HAS e consumo de bebidas alcóolicas, sendo que um paciente pode apresentar mais de um fator de risco. Nesse sentido, aqueles que apresentavam quatro ou mais desses fatores aumentavam em quatro vezes a chance de ter uma doença isquêmica do coração. O estudo foi conduzido com pacientes acima de 60 anos e, quase que na sua totalidade, observou-se maior prevalência de dietas inadequadas, sedentarismo, obesidade, tabagismo e consumo de álcool.

No estudo de Souza et al. (2020), no qual foram analisados 49 idosos e a média de idade foi de 71,5 anos, constatouse elevada prevalência de RCV e os fatores de risco mais prevalentes foram HAS, obesidade e sedentarismo, porém houve menor prevalência de tabagismo e de alcoolismo. Em relação à obesidade, existe uma forte relação com o RCV e os níveis aumentados de adiposidade. Dessa forma, tem sido maior a atenção às medidas antropométricas nos idosos, como o IMC, a CC e a RCE, a fim de avaliar a redistribuição de gordura devido ao aumento desproporcional na adiposo corporal e visceral. A importância dessa avaliação se dá pelo fato de que a obesidade abdominal visceral aumenta o RCV, pois o tecido adiposo facilita a entrada de ácidos graxos livres nos hepatócitos, aumentando o metabolismo lipídico e, consequentemente, o risco de desenvolvimento de dislipidemia.

Além disso, ainda de acordo com Souza et al. (2020), foi observada elevada frequência de HAS, que é uma doença de caráter silencioso, com fatores de risco cardíacos, renais e cardiovasculares, associada, principalmente, ao excesso de peso e à falta de atividade física. Isso se deve, em especial, às alterações fisiológicas, dificultando a realização de exercícios físicos. 
Como a HAS é uma condição em que os níveis pressóricos estão constantemente altos, ocorre a produção de lesão nas artérias de grande, médio e pequeno calibre, bem como no coração e em outros órgãos nobres, como cérebro e rins (Magalhães, Amorim \& Rezende, 2018).

Segundo Moretti et al. (2009), existe maior prevalência de dislipidemia mista em idosos obesos e do sexo feminino. Dos 99 idosos analisados, 70\% foram classificados como obesos e a maioria desses apresentou os níveis de lipoproteínas elevados, apresentando maior probabilidade de desenvolver doenças crônicas não transmissíveis. Pode-se dizer, portanto, que a população idosa apresenta aumento de peso corporal, o qual é consequência do próprio processo de envelhecimento, pois a composição corporal sofre alterações, apresentando redução da massa muscular e aumento do volume de tecido adiposo principalmente na região abdominal, o que está diretamente relacionado com o aumento da obesidade e de doenças secundárias nessa população. Esse aumento no peso corpóreo está relacionado com o maior risco para o desenvolvimento de doenças coronarianas, o que justifica adotar medidas preventivas e efetivas nessa população. Dessa forma, os cuidados primários, como exercícios físicos, dietas balanceadas e interrupção do alcoolismo e do tabagismo são a melhor alternativa para se evitarem tais agravos à saúde (Almeida et al., 2016).

Em consonância com os achados de Moretti et al. (2009), Gomez (2005) verificou que a dislipidemia mista em pacientes maiores de 60 anos foi mais prevalente nas mulheres do que nos homens. Além disso, as principais doenças associadas às dislipidemias foram HAS, diabetes mellitus e AVC. Os resultados obtidos por Souza et al. (2016) também corroboram com os encontrados por Moretti et al. (2009) e a justificativa para esse achado está relacionada à reposição hormonal e à menopausa, que são fatores que influenciam diretamente na desordem dos níveis lipídicos no sexo feminino. Os fatores de risco importantes para o aumento dos lipídios séricos são o sedentarismo, a obesidade, a idade e o sexo. É importante destacar que, dentre os óbitos causados por doenças no aparelho circulatório, as dislipidemias foram responsáveis por uma parcela significativa deles.

Reafirmando a maior incidência de níveis lipídicos elevados em mulheres idosas, Huang et al. (2005) observou que a prevalência de dislipidemia, considerando níveis elevados de colesterol total, LDL-c e triglicerídeos, nessa ordem, foi maior em mulheres do que em homens. Ademais, observa-se que essa condição é frequente naquelas socioeconomicamente desfavorecidas, ou seja, as condições socioeconômicas desfavoráveis e o estilo de vida influenciam na prevalência da dislipidemia (Melo \& Lima, 2020).

Diniz e Tavares (2013) relataram que, dos sete fatores de RCV utilizados em seu estudo (tabagismo, sedentarismo, obesidade, CA aumentada, HAS, diabetes mellitus e alcoolismo), seis foram mais prevalentes entre as mulheres, enquanto que nos homens apenas o alcoolismo se sobressaiu. Desse modo, observou-se a necessidade de implantar atividades físicas nas ESF, contando com a presença de um educador físico e de acompanhamento com uma nutricionista.

As idosas, portanto, são mais vulneráveis para os fatores de RCV, uma vez que elas apresentaram maior prevalência de medidas antropométricas elevadas, como IMC e CC, e de HAS, de hipercolesterolemia, de excesso de peso e de adiposidade central (Soar, 2015).

A maioria dos idosos com risco cardiovascular apresentou IMC, CC e níveis séricos de glicose acima dos valores de referência, que são, de acordo com a Diretriz da Sociedade Brasileira de Diabetes, respectivamente, $22-27 \mathrm{~kg} / \mathrm{m}^{2},<88 \mathrm{~cm}$ (para mulheres) ou $<102 \mathrm{~cm}$ (para homens) e $<100 \mathrm{mg} / \mathrm{dl}$. Verifica-se que as dislipidemias nos idosos são de origem secundária, pois estão associadas, principalmente, ao diabetes mellitus e à obesidade. Esses resultados corroboram com os de Ferreira et al. (2017) envolvendo fatores de risco para doenças cardiovasculares em idosos, no qual a prevalência de obesidade foi de 62 indivíduos em um total de 246, o que pode estar associado ao aumento do tecido adiposo no abdômen ocasionado pelo próprio processo de envelhecimento. 
Em relação ao estado civil, o estudo de Ferreira et al. (2017) mostrou que 48\% dos participantes eram casados ou viviam com companheiros e 34,6\% eram viúvos. Dessa maneira, ser casado consistiu em um fator essencial de proteção, pois o suporte familiar é extremamente importante tanto na aderência ao tratamento medicamentoso quanto ao tratamento não medicamentoso, a exemplo da prática de atividades físicas. Ademais, esse estudo estimou que $10 \%$ da população idosa consome álcool e isso é mais comum no sexo masculino. Além de elevar o RCV nesses indivíduos, o envelhecimento provoca alterações fisiológicas no organismo, o que atrapalha o metabolismo do álcool, deixando os idosos mais susceptíveis à intoxicação alcoólica.

No estudo de Garcez et al. (2014), os resultados revelaram que a população de idosos obesos apresenta uma porcentagem superior em relação à população de adolescentes obesos. Além disso, é comprovado que existe uma maior prevalência de inadequação de perfil lipídico em indivíduos com excesso de peso. Em relação à CC inadequada na população idosa, houve maior frequência de hipertrigliceridemia isolada e HDL-c baixo. Diante disso, cabe ressaltar que, no Brasil, o número de mortes ocasionadas por doenças cardiovasculares está apresentando um aumento principalmente devido ao IMC elevado.

O aumento de gordura corporal nos idosos é um fator que determina o aumento de doenças relacionadas ao aparelho cardiovascular (Santos et al., 2013). Segundo Rocha et al. (2013), com o envelhecimento, ocorrem mudanças no organismo que favorecem o aparecimento de distúrbios subsequentes que influenciam no aumento do RCV. Dentre essas mudanças, o acúmulo de gordura, principalmente na região abdominal, favorece o aparecimento de dislipidemias, as quais contribuem para a intensificação do RCV (Rezende Et al., 2006).

Nessa perspectiva, os resultados obtidos no estudo de Cruz et al. (2004) demonstraram que nos homens idosos considerados obesos houve associação entre hipercolesterolemia e obesidade e eles apresentaram pressão arterial diastólica e níveis plasmáticos de colesterol total e LDL-c mais elevados. Nas idosas obesas, a pressão arterial sistólica e os níveis plasmáticos de glicose foram mais elevados e os níveis de HDL-c foram menores. Já a hipertrigliceridemia foi maior em ambos os sexos.

Além disso, é possível visualizar a maior presença de HAS na população idosa porque essa doença segue um padrão direto e linear conforme a idade avança. No Brasil, mais de $60 \%$ desses indivíduos apresentam elevação patológica da pressão arterial e esse fator contribui de maneira direta ou indireta para 50\% das mortes consequentes a doenças cardiovasculares (Magalhães, Amorim \& Rezende, 2018). A disfunção endotelial, doença na qual há redução da produção de óxido nítrico, e a maior sensibilidade à angiotensina II, consequente ao aumento dos receptores angiotensinérgicos 1 nas células da musculatura lisa, são aspectos fisiopatológicos envolvidos na gênese da HAS e da dislipidemia que correlacionam essas duas doenças (Marte \& Santos, 2007).

\section{Considerações Finais}

Conclui-se que existe relação entre a dislipidemia mista e a evolução de doenças cardiovasculares em idosos. As principais causas comportamentais para esses dois fatores são, nessa ordem, a obesidade, o sedentarismo, a alimentação inadequada, o tabagismo e a ingestão de bebidas alcoólicas. Além disso, por mais que não seja uma causa comportamental, grande parte dos artigos demonstraram que a doença está associada ao gênero feminino.

\section{Referências}

Agência IBGE Notícias (2018). Projeção da População 2018: número de habitantes do país deve parar de crescer em 2047. https://agenciadenoticias.ibge.gov.br/agencia-sala-de-imprensa/2013-agencia-de-noticias/releases/21837-projecao-da-populacao-2018-numero-de-habitantesdo-pais-deve-parar-de-crescer-em-2047\#: :text=A\%20popula\%C3\%A7\%C3\%A3o\%20total\%20projetada\%20para,(228\% 2C4\%20milh\%C3\%B5es). 
Almeida, F. S., Silva, K. S. M., Silvino, M. R. S., Damacena, D. O., \& Pereira, H. S. (2016). Análise do Perfil Lipídico e Risco Cardiovascular em Idosos. Congresso Brasileiro de Ciências da Saúde. http://www.editorarealize.com.br/artigo/visualizar/18886.

Arquivos Brasileiros de Cardiologia: Atualização da Diretriz Brasileira de Dislipidemias e da Prevenção da Aterosclerose (2017). Revista da Sociedade Brasileira de Cardiologia, 109(2), 1-76. https://www.scielo.br/pdf/abc/v109n2s1/0066-782X-abc-109-02-s1-0001.pdf.

Azzi, J. B., Vale, I. G., \& Silva, R. M. (2018). Utilização de máquina de vetor de suporte na caracterização de pacientes portadores de doenças cardiovasculares. Proceeding Series of the Brazilian Society of Applied and Computational Mathematics, 6(1), 1-2. https://webcache.googleusercontent.com/search?q=cache:ByZVcL0x7QcJ:https://proceedings.sbmac.org.br/sbmac/article/download/1977/1995+\&cd=1\&hl=pt $-\mathrm{BR} \& \mathrm{ct}=\mathrm{clnk} \& \mathrm{gl}=\mathrm{br}$.

Borim, F. S. A., Francisco, P. M. S. B., \& Neri, A. L. (2017). Fatores sociodemográficos e de saúde associados à mortalidade em idosos residentes na comunidade. Revista Saúde Pública, 51(42), 1-12. 10.1590/s1518-8787.2017051006708.

Cruz, I. B. M., Almeida, M. S. C., Schwanke, C. H. A., \& Moriguchi, E. H. (2004). Prevalência de obesidade em idosos longevos e sua associação com fatores de risco e morbidades cardiovasculares. Revista da Associação Médica Brasileira, 50(2), 172-177. 10.1590/S0104-42302004000200034.

Diniz, M. A., \& Tavares, D. M. S. (2013). Fatores de Risco para Doenças Cardiovasculares em Idosos de um Município do Interior de Minas Gerais. Texto Contexto Enfermagem, 22(4), 885-892. 10.1590/S0104-07072013000400003.

Ferreira, J. D. F., Moreira, R. P., Maurício, T. F., Lima, P. A., Cavalcanti, T. F., \& Costa, E. C. (2017). Fatores de Risco para Doenças Cardiovasculares em Idosos. Revista de Enfermagem, 11(12), 4895-4905. 10.5205/1981-8963-v11i12a15182p4895-4905-2017.

Fontana, L., Addante, F., Copetti, M., Paroni, G., Fontana, A., Sancarlo, D.,Pellegrini, F., Ferrucci, L., \& Pilotto, A. (2013). Identification of a metabolic signature for multidimensional impairment and mortality risk in hospitalized older patients. Aging Cell, 12(3), 459-466. $10.1111 /$ acel.12068

Garcez, M. R., Pereira, J. L., Fontanelli, M. M., Marchioni, D. M. L., \& Fisberg, R. M. (2014). Prevalence of Dyslipidemia According to the Nutritional Status in a Representative Sample of São Paulo. Arquivos Brasileiros de Cardiologia, 103(6), 476-484. $10.5935 / \mathrm{abc} .20140156$

Gomez, J. A. C. (2005). Dislipidemia en ancianos. Anales de la Facultad de Ciencias Médicas (Assunción), 38(1-2), 63-67. http://scielo.iics.una.py/scielo.php?script=sci_arttext\&pid=S1816-89492005000100007.

Huang, ,K. Lee, M. S., Lee, S. D., Chang Y. H., Lin, Y. C., Tu S. H., \& Pan, W. H. (2005). Obesity in the Elderly and its Relationship with Cardiovascular Risk Factors in Taiwan. Obesity Research, 13(1), 170-178. 10.1038 / oby.2005.22.

Kopin, L., \& Lowenstein, C. (2017). Dyslipidemia. Annals of Internal Medicine, 167(11), 81-96. 10.7326 / AITC201712050.

Lotufo, P. A., Santos, R. D., Sposito, A. C., Bertolami, M., Neto, J. R. F., Izar, M. C., Szwarcwald, C., Prado, R. R., Stoppa, S. R., Malta, D. C., \& Bensenor I. M. (2017). Self-Reported High-Cholesterol Prevalence in the Brazilian Population: Analysis of the 2013 National Health Survey. Arquivo Brasileiro de Cardiologia, 108(5), 411-416. 10.5935/abc.20170055

Magalhães, L. B. N. C., Amorim, A. M., \& Rezende, E. P. (2018). Conceitos e Aspectos Epidemiológicos da Hipertensão Arterial. Revista Brasileira de Hipertensão, 25(1), 6-12. http://departamentos.cardiol.br/sbc-dha/profissional/revista/25-1.pdf.

Marte, A. P., \& Santos, R. D. (2007). Bases fisiopatológicas da dislipidemia e hipertensão arterial. Revista Brasileira de Hipertensão, 14(4), 252-257. http://departamentos.cardiol.br/dha/revista/14-4/09-fisiopatologicas.pdf.

Martins, M. V., Souza J. D., Martinho K. O., Franco F. S., \& Tinôco A. L. A. (2017). Association between triglycerides and HDL-cholesterol ratio and cardiovascular risk factors among elderly persons receiving care under the family health strategy of Viçosa, Minas Gerais. Revista Brasileira de Geriatria e Gerontologia, 20(2), 236-243. 10.1590/1981-22562017020.160059.

Melo, L. A., \& Lima, K. C. (2020). Fatores associados às multimorbidades mais frequentes em idosos brasileiros. Ciência \& Saúde Coletiva, 25(10), 38793888. 10.1590/1413-812320202510.35632018.

Moretti, T., Moretti, M. P., Moretti, M., Sakae T. M., Sakae, D. Y., \& Araújo, D. (2009). Estado nutricional e prevalência de dislipidemias em idosos. Arquivos Catarinenses de Medicina, 38(3), 12-16. http://www.acm.org.br/acm/revista/pdf/artigos/747.pdf.

Nagatsuyu, D. T., Moriguti, E. K. U., Pfrimer, K., Formighieri, P. F., Lima, N. K. C., Ferriolli, E., \& Moriguti, J. C. (2009). O impacto da obesidade abdominal sobre os níveis plasmáticos de lipídeos nos idosos. Revista Faculdade de Medicina Usp, 42(2), 157-163. 10.11606/issn.2176-7262.v42i2p157-163.

Olubamwo, O. O., Virtanem, J. K., Voutilainen, A., Kauhanen, J., Pihlajamaki, J., \& Tuomainen, T. P. (2018). Association of fatty liver index with the risk of incident cardiovascular disease and acute myocardial infarction. European Journal Of Gastroenterology \& Hepatology, 30(9), 1047-1054. 10.1097 / MEG.0000000000001183.

Pereira, J. C., Barreto, S. M., \& Passos, V. M. A. (2008). O Perfil de Saúde Cardiovascular dos Idosos Brasileiros Precisa Melhorar: Estudo de Base Populacional. Arquivos Brasileiros de Cardiologia, 91(1), 1678-4170. 10.1590/S0066-782X2008001300001.

Pereira, A. S. et al. (2018). Metodologia da pesquisa científica. UFSM. https://www.ufsm.br/app/uploads/sites/358/2019/02/Metodologia-da-PesquisaCientifica_final.pdf.

Prince, M. J., Wu, F., Guo, Y., Robledo, L. M. G., O'Donnell, M., Sullivan, R., \& Yusuf, S. (2014). The burden of disease in older people and implications for health policy and practice. The Lancet, 385(9967), 549-562. 10.1016/S0140-6736(14)61347-7. 
Research, Society and Development, v. 10, n. 3, e38310313416, 2021

(CC BY 4.0) | ISSN 2525-3409 | DOI: http://dx.doi.org/10.33448/rsd-v10i3.13416

Rezende, F. A. C., Rosado, L. E. F. P. L., Ribeiro, R. C. L., Vidigal, F. C., Vasques, A. C. J., Bonard, I. S., \& Carvalho, C. R (2006). Índice de massa corporal e circunferência abdominal: associação com fatores de risco cardiovascular. Arquivos Brasileiros de Cardiologia, 87(6), 728-734. 10.1590/S0066$782 X 2006001900008$.

Rocha, F. L., Menezes, T. N., Melo, R. L P., \& Pedraza, D. F. (2013). Correlação entre indicadores de obesidade abdominal e lipídeos séricos em idosos. Revista Associação Médica Brasileira, 59(1), 48-55. 10.1590/S0104-42302013000100011.

Santos, R. R., Bicalho, M. A. C., Mota, P., Oliveira, D. R., \& Moraes, E. N. (2013). Obesidade em idosos. Revista Médica de Minas Gerais, 23(1), 64-73. $10.5935 / 2238-3182.20130011$.

Schatz, I. J., Masaki, K., Yano, K., Chen, R., Rodriguez, B. L., \& Curb, J. D. (2001). Cholesterol and all-cause mortality in elderly people from the Honolulu Heart Program: a cohort study. Lancet, 358(9279), 351-355. 10.1016 / S0140-6736 (01) 05553-2.

Soar, C. (2015). Prevalência de fatores de risco cardiovascular em idosos não institucionalizados. Revista Brasileira de Geriatria e Gerontologia, 18(2), 385395. 10.1590/1809-9823.2015.14072.

Sociedade Brasileira de Diabetes: Diretrizes Sociedade Brasileira de Diabetes (2019-2020). Sociedade Brasileira de Diabetes, 12-489. https://www.diabetes.org.br/profissionais/images/DIRETRIZES-COMPLETA-2019-2020.pdf.

Souza, A. M., Pereira, M. H. Q., Teles, B. K. A., Souza, A. L., Pereira, D. S., \& Pereira M. L. A. S. (2020). Marcadores de risco cardiovascular em idosos da Estratégia de Saúde da Família. Brazilian Journal of Development, 6(6), 36094-36109. 10.34117/bjdv6n6-234.

Souza, F. O., Júnior, C. Q. L., Siqueira, I. C., Oliveira, N. C., Tavares, R. S., Rocha, T. M. D., \& Moura, A. L. D. (2016). Avaliação do perfil lipídico de pacientes acima de 60 anos de idade atendidos em um laboratório-escola. Revista Brasileira de Análises Clínicas, 48(2), 70-75. 10.21877/24483877.201600452 .

Zaslavsky, C., \& Gus, I. (2002). Idoso: Doença Cardíaca e Comorbidades. Arquivos Brasileiros de Cardiologia, 79(6), 635-639. http://publicacoes.cardiol.br/abc/2002/7906/79060011.pdf. 